\title{
Factores familiares y sociales asociados al bienestar emocional en adolescentes diabéticos
}

\author{
C. de Dios, MD \\ C. Avedillo, MD \\ A. Palao, MD \\ A. Ortiz, MD \\ J.L. Agud, MD
}

Servicio de Psiquiatría, Hospital Universitario La Paz, Madrid; y Servicio de Medicina Interna, Hospital Severo Ochoa, Leganés, Madrid

ESPAÑA

\begin{abstract}
RESUMEN - Hemos realizado un estudio transversal en el que han participado 55 adolescentes diabéticos y sus familias. Se analizan las relaciones existentes entre factores sociodemográficos, características generales de la familia, apoyo específico a la enfermedad, bienestar del adolescente y control metabólico. El sexo y la cohesión familiar son los factores que con más fuerza predicen el bienestar del adolescente diabético. El apoyo específico a la enfermedad tanto por parte de la familia como de los amigos es también un importante factor asociado al bienestar. No se encuentra ninguna asociación entre las variables estudiadas y el control metabólico. Los resultados nos llevan a la conclusión de que, en los adolescentes estudiados, el bienestar emocional depende de factores personales y de su entorno, y es independiente del buen control de su glucemia.
\end{abstract}

\section{Introducción}

El estudio y la mejora de la calidad de vida de los pacientes es en la actualidad un objetivo central en la asistencia sanitaria (Guyatt, Feeny et al. 1993), siendo cada vez mayor el número de estudios que se publican sobre el tema, así como la importancia que se le concede en la evaluación de la respuesta al tratamiento y la evolución de las enfermedades.
Aunque no existe acuerdo suficiente sobre la definición de calidad de vida, en la mayor parte de las definiciones se consideran el bienestar y la satisfacción subjetivos como partes de la misma (Katschnig, Freeman et al. 2000). Otros autores diferencian calidad de vida de estado de salud, y en este caso el bienestar sería un indicador del estado de salud emocional (Bradley 2001).

El paciente con diabetes mellitus se ve sometido a lo largo de su enfermedad a dife- 
rentes situaciones -el diagnóstico inicial, las demandas que la enfermedad le plantea en su vida diaria, la aparición de posibles complicaciones-, que repercuten de manera importante en su bienestar físico, psicológico y social (Stewart, Greenfield et al. 1989), (Cox y Gonder-Frederick 1992), (Bradley y Gamsu 1994). En la literatura reciente destacan estudios sobre la evaluación de la calidad de vida en los pacientes diabéticos tipo I (1988), (Ingersoll y Marrero 1991), (Bott, Muhlhauser et al. 1998), (Bradley y Gamsu 1994), (Golden 1998), calidad de vida y tipo de tratamiento (1996), (Grey y Boland et al. 1998), (Grey y Boland et al. 2000), calidad de vida y factores psicosociales (Skinner y Hampson 1998), (Skinner y John et al. 2000), demográficos (Eiser y Flynn et al. 1992) y otros factores (Glasgow y Ruggiero et al. 1997), (Petterson y Lee et al. 1998), (de Sonnaville y Snoek et al. 1998), (Wu y Sainfort et al. 1998).

Diversos autores han estudiado la relación entre la adherencia al tratamiento, el control metabólico y los factores familiares (Burroughs, Harris et al. 1997), (Drotar 1997) y sociales (Greca, Auslander et al. 1995). Hay menos estudios que analicen estos factores y su influencia sobre la calidad de vida (Skinner y Hampson 1998; Skinner, John et al. 2000; Skinner y Hampson 2001), (Guttmann-Bauman, Flaherty et al. 1998), (Grey, Boland et al. 1998). Una red social y familiar adecuadas promueven el bienestar emocional y el sentimiento de ser valorado y cuidado por otros, aspectos importantes para una adecuada adaptación a la enfermedad (Greca 1992; Greca, Auslander et al. 1995) (Resnick, Bearman et al. 1997). El objetivo de nuestro trabajo es analizar qué factores del medio familiar y social se asocian a mayor o menor bienestar emocional en los adolescentes diabéticos. Hasta donde conocemos, sólo el trabajo de
Grey 1998 (Grey, Boland et al. 1998) ha estudiado en parte estos aspectos. Nuestro trabajo analiza tanto factores generales del medio familiar y social como factores de apoyo específico a la diabetes y su influencia sobre el bienestar.

\section{Material y método}

Se invitó a participar en el estudio a todos los pacientes adolescentes (12-19 años) en seguimiento en las consultas de diabetología del Hospital La Paz (Madrid), así como a sus padres. La invitación se realizó a través de reuniones, por carta y en las consultas. El estudio se plantea como un seguimiento de las familias en un período de dos años, siendo el que presentamos aquí el primer corte transversal que realizamos.

Tanto el adolescente como los padres han dado su consentimiento por escrito para la realización del estudio, que ha sido asimismo aprobado por la comisión ética del Hospital. A todos ellos se les ofrecieron obsequios en reconocimiento a su colaboración.

Se excluyeron 12 pacientes por no cumplir los criterios de inclusión (DMID de al menos un año de evolución, en seguimiento regular en las consultas, no otra enfermedad crónica grave salvo patología tiroidea controlada). De los 96 que cumplen los criterios de inclusión, participan el 57\%. No hay diferencias estadísticamente significativas entre los que participan (tabla I) y los que no en cuanto a parámetros sociodemográficos y médicos (control metabólico, duración de la enfermedad, edad al diagnóstico, número de hipoglucemias y hospitalizaciones en los últimos seis meses, complicaciones metadiabéticas, número de pinchazos de insulina). 
Tabla I

Características sociodemográficas de los participantes

\begin{tabular}{|c|c|c|}
\hline & $\mathrm{N}$ & $\%$ \\
\hline \multicolumn{3}{|l|}{ Sexo } \\
\hline Varones & 33 & 60 \\
\hline Mujeres & 22 & 40 \\
\hline \multicolumn{3}{|l|}{ Nivel socioeconómico } \\
\hline Bajo & 13 & 27,1 \\
\hline Medio & 26 & 54,2 \\
\hline Alto & 9 & 18,8 \\
\hline \multicolumn{3}{|l|}{ Historia conyugal } \\
\hline Unión estable & 47 & 90,4 \\
\hline Separación, divorcio, orfandad & 5 & 9,6 \\
\hline \multicolumn{3}{|l|}{ Con quién convive } \\
\hline Con padre y madre & 48 & 90,6 \\
\hline Con madre & 4 & 7,5 \\
\hline Otros & 1 & 1,9 \\
\hline \multicolumn{3}{|l|}{ Estadio de Tanner } \\
\hline Púberes y prepúberes (1-4) & 19 & 34,5 \\
\hline Pospúberes (5) & 36 & 65,5 \\
\hline \multicolumn{3}{|l|}{ Número de pinchazos/24h } \\
\hline 2 & 5 & 9,6 \\
\hline 3 & 44 & 84,6 \\
\hline 4 & 3 & 5,7 \\
\hline Edades (años) & Media & D.E. \\
\hline Edad paciente & 16,9 & 2,17 \\
\hline Edad madre & 45,5 & 7,24 \\
\hline Edad padre & 49,2 & 5,3 \\
\hline Duración enfermedad (años) & 7,2 & 4,13 \\
\hline Media $\mathrm{HbA}_{1 \mathrm{C}}$ & $8,30 \%$ & 1,2 \\
\hline
\end{tabular}

Los participantes fueron asesorados acerca de cómo completar los cuestionarios aplicados, que hicieron de manera individual. Se les comunicó además que los datos proporcionados no serían conocidos por los equipos respectivos de los Servicios de Endocrinología.

\section{Descripción de los cuestionarios}

Cuestionario de Bienestar W-BQ22 (Bradley 1994): se diseñó en origen para ser aplicado en pacientes con diabetes, y evalúa respuestas emocionales como depresión, ansiedad, energía y bienestar. La versión que hemos utilizado consta de 22 ítems, que se evalúan en una escala de Likert de 0-3, a partir de los cuales se calculan cuatro subes- calas: depresión, ansiedad, energía y bienestar positivo. Mayor puntuación en cada una de las subescalas indica que el individuo presenta de manera más intensa la respuesta emocional que se evalúa. Mediante la suma de la puntuación corregida y ajustada en cada una de estas subescalas se obtiene la puntuación de bienestar general, que permite una puntuación máxima de 66. La consistencia interna medida por el coeficiente alfa de Cronbach es (Bradley 1994) de 0,67 (depresión); 0,74 (ansiedad); 0,64 (energía) y 0,80 (bienestar positivo).

Escala de Clima Social en la Familia (Moos 1974): Evalúa las relaciones interpersonales entre los miembros de la familia, la dirección marcada desde el seno familiar 
para el crecimiento personal, y su estructura organizativa básica. (Moos y Moos 1976) Se ha utilizado la versión validada en español (Moos, Moos et al. 1995)

Cuestionario de apoyo al cuidado diabético (CACDI) [32].Evalúa en qué medida la familia apoya y se implica en el buen manejo de la diabetes (dieta, ejercicio etc.), en cuestionarios individualizados para recoger la percepción del paciente (CACDI hijo) y de cada uno de los progenitores (CACDI padres). En su forma para el adolescente, la prueba es unifactorial, y en el estudio de validación los autores dan una fiabilidad medida por el a de Cronbach de 0,97 (Rangos posibles de puntuación: 73-219). La forma P (CACDI padres) presenta dos factores. El Factor 1 se denomina "conductas de apoyo familiar" y el Factor 2, "conductas de no apoyo". Respecto a la consistencia interna, el Factor 1 presenta un coeficiente $\mathrm{a}=0,93$, y el Factor 2 un coeficiente $\mathrm{a}=$ 0,91. La fiabilidad para el cuestionario en su totalidad es de 0,87. En nuestro estudio, hemos considerado el promedio de las respuestas del padre y las de la madre, al tener ambos cuestionarios un coeficiente de correlación intraclase de 0,78 (conductas de apoyo) y de 0,85 (conductas de no apoyo). (Rangos posibles de puntuación: 0-192).

El cuestionario de apoyo de los profesionales (CACDI p. sanitario) es unifactorial, y presenta un coeficiente $a=0,94$. (Rangos posibles de puntuación: 26-78).

El coeficiente a para el cuestionario de apoyo de los amigos (CACDI amigos) es de 0,94 (Rangos posibles de puntuación: 34-102).

\section{Control metabólico}

Se ha considerado la media de las cifras de hemoglobina glicada $\left(\mathrm{HbA}_{1 \mathrm{c}}\right)$ de los seis meses inmediatamente anteriores a la fecha en que se completaron los cuestionarios. La determinación se ha realizado mediante cromatografía de alta resolución (Bio-Rad, Richmon, CA) (Valores normales 4,7-5,8\%).

\section{Análisis estadístico}

Análisis univariante: comparación de medias, prueba T de Student y pruebas no paramétricas, según caso. Prueba ANOVA entre variables numéricas y categóricas de más de dos categorías o, en su caso, test de Kruskal-Wallis. Entre variables numéricas se han aplicado coeficientes de correlación de Pearson o de Spearman. Tablas de contingencias (cualixcuali) con prueba de $\chi^{2}$, test exacto de Fisher o de McNemar.

Posteriormente se ha realizado análisis de regresión jerárquica múltiple, en el que se han introducido como variables respuesta todas las subescalas del cuestionario de bienestar y como variables independientes aquellas que hemos pensado, sobre la base del análisis previo, que podrían predecir la respuesta. Así, se han introducido en primer lugar las variables sociodemográficas y de maduración, y posteriormente las distintas dimensiones de la escala de Moos, la puntuación en los cuestionarios de apoyo específico de la familia, de los profesionales y de los amigos.

En todas las pruebas hemos tomado como valores significativos $\mathrm{p}<0,05$.

Se ha utilizado el paquete estadístico SPSS.

\section{Resultados}

\section{Bienestar y variables sociodemográficas}

El sexo femenino se asocia a mayor puntuación en las subescalas de depresión y 
ansiedad, y menor en las de energía y bienestar positivo. Globalmente, las adolescentes diabéticas manifiestan menos bienestar general. Estas asociaciones se mantienen al corregir para la edad.

Los adolescentes más jóvenes manifiestan más bienestar positivo que los mayores; si consideramos el estadio de Tanner, los prepúberes y púberes presentan menos ansiedad y mayor puntuación en las escalas de bienestar positivo, energía y bienestar general que los pospúberes.

No hay diferencias estadísticamente significativas en relación con la duración de la enfermedad, la edad al diagnóstico, el nivel educativo o el socioeconómico, pero sí en cuanto a la historia conyugal. Así, los adolescentes que viven en familias rotas o monoparentales manifiestan más depresión y menos bienestar general.

Tabla II

Bienestar según características sociodemográficas

\begin{tabular}{|c|c|c|c|c|c|}
\hline & $\begin{array}{c}\text { Depresión } \\
\text { Rango: 0-18 } \\
\text { (media, D.S.) }\end{array}$ & $\begin{array}{c}\text { Ansiedad } \\
\text { Rango: 0-18 } \\
\text { (media, D.S.) }\end{array}$ & $\begin{array}{c}\text { Energía } \\
\text { Rango: 0-12 } \\
\text { (media, D.S.) }\end{array}$ & $\begin{array}{l}\text { B. positivo } \\
\text { Rango: 0-18 } \\
\text { (media, D.S.) }\end{array}$ & $\begin{array}{c}\text { B. general } \\
\text { Rango: 0-66 } \\
\text { (media, D.S.) }\end{array}$ \\
\hline \multicolumn{6}{|l|}{ Sexo } \\
\hline Varones & $3,1(2,8)^{* * * *}$ & $2,9(3,1) * * *$ & $10,4(1,6)^{* * *}$ & $14,5(3,0) * * *$ & $54,8(8,6)^{* * *}$ \\
\hline Mujeres & $6,8(3,7)$ & $7,8(3,2)$ & $7,1(2,5)$ & $9,6(4,4)$ & $38,1(10,9)$ \\
\hline \multicolumn{6}{|l|}{ Edad } \\
\hline$<=16$ & $3,8(3,0)$ & $3,9(3,5)$ & $9,8(2,9)$ & $14,6(3,4)^{*}$ & $52,7(10,3)$ \\
\hline$>16$ & $4,9(3,8)$ & $5,2(4,1)$ & $8,8(2,4)$ & $11,7(4,4)^{*}$ & $46,2(13,0)$ \\
\hline \multicolumn{6}{|l|}{ Tanner } \\
\hline Prepúberes y púberes & $3,8(3,0)$ & $3,1(3,6)^{*}$ & $10,3(1,5)^{*}$ & $14,3(3,9)^{*}$ & $53,7(9,3)^{*}$ \\
\hline Pospúberes & $5,0(3,9)$ & $5,7(3,8)$ & $8,4(2,8)$ & $11,7(4,3)$ & $45,4(13,2)$ \\
\hline \multicolumn{6}{|l|}{ Nivel socioeconómico } \\
\hline Bajo & $5,(4,0)$ & $5,7(4,3)$ & $9,2(2,6)$ & $11,1(4,9)$ & $45,5(14,4)$ \\
\hline Medio & $4,4(4,1)$ & $4,4(4,0)$ & $9,1(2,5)$ & $13,5(3,9)$ & $49,7(13,0)$ \\
\hline Alto & $4,5(2,7)$ & $4,6(4,2)$ & $8,6(3,3)$ & $11,8(4,8)$ & $47,3(11,8)$ \\
\hline \multicolumn{6}{|l|}{ Historia conyugal } \\
\hline Unión estable & $4,1(3,5)^{* *}$ & $4,4(3,8)$ & $9,3(2,5)$ & $12,8(4,3)$ & $49,5(12,3) *$ \\
\hline Separación y otros & $9,0(2,3)$ & $8,2(4,1)$ & $7,4(2,7)$ & $11,4(4,9)$ & $37,6(12,1)$ \\
\hline
\end{tabular}

(*) $\mathrm{p}=<0,05$.

$(* *) \mathrm{p}=<0,01$.

$(* * *) \mathrm{p}=<0,001$.

\section{Bienestar y variables médicas}

No hay asociaciones significativas entre la media de $\mathrm{HbA}_{1 \mathrm{c}}$ y ninguna de las escalas de bienestar.

Si consideramos el número de inyecciones diarias de insulina, a más pinchazos los pacientes manifiestan menos energía ( $\mathrm{r}=$ $0,287, \mathrm{p}<0,05)$.

\section{Bienestar y medio familiar (Escala de Moos)}

En estas familias no hay asociaciones significativas entre las dimensiones de la escala Moos y las variables sociodemográficas (edad, tamaño familiar, nivel socioeconómico, historia conyugal).

Cuando consideramos la familia en su conjunto, observamos dos diferencias res- 
pecto al promedio de las familias españolas (tabla III). Las familias con un hijo diabético parecen ser menos "autónomas". Esta dimensión mide el grado en que los miembros de la familia están seguros de sí mismos, son autosuficientes y toman sus propias decisiones (Moos 1974). Asimismo muestran una tendencia a presentar una estructura más rígida, con reglas y procedimientos establecidos.

No se detectan diferencias estadísticamente significativas en cuanto a los valores centrales del índice de incongruencia (IF).
Es decir, no parece que haya mayor discrepancia interna en estas familias que en una familia media española.

El adolescente con mayor bienestar general es aquel que percibe a su familia como más cohesionada y organizada, con bajo nivel de conflicto. No contribuye a su bienestar el que haya demasiada autonomía, ni tampoco que la familia esté fuertemente orientada a la consecución de objetivos o logros.

Los más deprimidos y ansiosos perciben a sus familias como más conflictivas y menos cohesionadas, con elevada autonomía y

Tabla III

Escala de FES en familias de adolescentes diabéticos y en la población española de validación

\begin{tabular}{|c|c|c|c|}
\hline \multirow[t]{2}{*}{ Dimensiones } & \multirow{2}{*}{$\begin{array}{l}\text { F. diabéticos. }(\mathrm{N}=55) \\
\text { IF: } 17,9 \\
\text { Media }\end{array}$} & \multicolumn{2}{|c|}{$\begin{array}{l}\text { F. validación FES }(\mathrm{N}=346) \\
\text { IF: } 17,4\end{array}$} \\
\hline & & Media & $\mathrm{p}$ \\
\hline Cohesión & 6,4 & 6,6 & 0,5 \\
\hline Expresividad & 5,2 & 5,5 & 0,1 \\
\hline Conflicto & 3,4 & 3,1 & 0,2 \\
\hline Autonomía & 4,5 & 5,7 & 0,001 \\
\hline Consecución de logros & 5,6 & 5,6 & 0,8 \\
\hline Intelectual-cultural & 4,5 & 4,7 & 0,5 \\
\hline Social-recreativo & 4,5 & 4,3 & 0,4 \\
\hline Moralidad-religiosidad & 4,1 & 4,2 & 0,9 \\
\hline Organización & 6,1 & 6,2 & 0,8 \\
\hline Control & 4,5 & 4,1 & 0,09 \\
\hline
\end{tabular}

Tabla IV

Bienestar según FES (adolescentes; $\mathrm{n}=52$ )

\begin{tabular}{|c|c|c|c|c|c|}
\hline & Depresión & Ansiedad & Energía & $\begin{array}{c}\text { Bienestar } \\
\text { positivo }\end{array}$ & $\begin{array}{c}\text { Bienestar } \\
\text { general }\end{array}$ \\
\hline Cohesión & $-0,4 * * *$ & $-0,3^{*}$ & $0,4 * * *$ & $0,5 * * * *$ & $0,4 * * *$ \\
\hline Expresividad & $-0,06$ & $-0,09$ & 0,1 & 0,1 & 0,1 \\
\hline Conflicto & $0,3 * *$ & $0,4 * * *$ & $-0,4 * * * *$ & $-0,4 * * * *$ & $-0,5 * * * *$ \\
\hline Autonomía & $0,4 * *$ & 0,2 & $-0,3 * * *$ & $-0,3 * *$ & $-0,3 * *$ \\
\hline Logros & $0,3 *$ & $0,2 *$ & $-0,2$ & $-0,2$ & $-0,3 * *$ \\
\hline Intelectual/cultural & $-0,1$ & 0,02 & 0,2 & 0,1 & 0,1 \\
\hline Social/recreativo & $-0,1$ & $-0,1$ & 0,2 & 0,04 & 0,1 \\
\hline Moral/religioso & $-0,1$ & $-0,1$ & 0,1 & 0,2 & 0,2 \\
\hline Organización & $-0,2 *$ & $-0,2 *$ & 0,2 & $0,2 *$ & $0,3^{*}$ \\
\hline Control & 0,05 & 0,1 & $-0,07$ & $-0,01$ & $-0,08$ \\
\hline
\end{tabular}

(*) $\mathrm{p}<=0,05$.

$(* *) \mathrm{p}<=0,01$.

$(* * *) \mathrm{p}<=0,001$.

$(* * * *) \mathrm{p}<=0,0001$. 
Tabla V

Bienestar y apoyo específico a la enfermedad (Coeficiente de Correlación de Spearman)

\begin{tabular}{lccccccccr} 
& N & \multicolumn{2}{c}{ Depresión Ansiedad Energía } & $\begin{array}{c}\text { Bienestar } \\
\text { positivo }\end{array}$ & $\begin{array}{c}\text { Bienestar } \\
\text { General }\end{array}$ & $\begin{array}{l}\text { CACDI CACDI } \\
\text { (hijo) }\end{array}$ \\
& & & & & $\begin{array}{l}\text { C. sanitario) } \\
\text { CACDI }\end{array}$ \\
\hline CACDI (hijo) & 48 & $-0,313^{*}$ & $-0,166$ & 0,239 & $0,542^{* *}$ & $0,367^{*}$ & & & 0,218 \\
CACDI (f.+) & 46 & $-0,296^{*}$ & $-0,04$ & 0,245 & $0,415^{* *}$ & 0,283 & $0,484^{* *}$ & $0,401^{* *}$ & 0,287 \\
CACDI (f.-) & 46 & 0,046 & 0,239 & $-0,084$ & $-0,119$ & $-0,154$ & 0,123 & 0,255 & $-0,056$ \\
CACDI & 48 & $-0,23$ & $-0,144$ & $0,305^{*}$ & $0,444^{* *}$ & $0,318^{*}$ & $0,526^{* *}$ & & 0,277 \\
CACDI & 48 & $-0,123$ & 0,039 & 0,066 & 0,256 & 0,108 & 0,218 & 0,277 & \\
\hline
\end{tabular}

(*) $\mathrm{p}<0,05$.

$(* *) \mathrm{p}<0,01$.

CACDI: Cuestionario de apoyo al cuidado diabético.

f.+: Conductas de apoyo.

f.-: Conductas de no apoyo.

orientadas predominantemente a lograr objetivos (y esto último se repite cuando se considera la percepción de la madre).

Los adolescentes que se sienten con más energía, manifiestan mayor cohesión en sus familias, menor nivel de conflicto y menos autonomía. Mientras que los que puntúan más alto en la escala de bienestar positivo, pertenecen también a familias más cohesionadas, con menos conflicto, menos autonomía y mayor grado de organización.

En nuestro trabajo, no se encuentran asociaciones significativas entre el grado de expresividad o de control en la familia y el bienestar del adolescente.

\section{Bienestar y Apoyo Específico a la enfermedad}

\section{Bienestar y Apoyo de la familia}

Tomándolos como grupo, parecen sentirse poco apoyados por parte de la familia (media 146,9, D.S. 39,8). Los menores de 16 años perciben más apoyo que los mayores $(\mathrm{p}=0,039)$, pero sigue siendo bastante inferior a los resultados obtenidos en una amplia muestra de diabéticos españoles de edad similar ([32]). En éstos, el percentil 50 se situaba entre 191-202, mientras que en nuestro estudio el percentil 50 está en 173 (134,5 para los mayores de 16 años).

$\mathrm{Si}$ consideramos la percepción del paciente del apoyo específico a su enfermedad que le presta su familia, encontramos que hay asociaciones positivas significativas entre apoyo percibido, bienestar positivo y bienestar general. Además, a menor apoyo percibido el paciente se siente más deprimido.

Respecto al apoyo expresado por los padres, vemos que cuanto mayor es el apoyo positivo que ofrecen, el hijo se muestra menos deprimido y con más bienestar positivo.

Hay una buena correlación entre el apoyo percibido por los hijos y el que los padres manifiestan dar (factor positivo).

Bienestar, apoyo de los amigos y del equipo sanitario

No hay diferencias estadísticamente significativas en cuanto a la edad del adolescente y la duración de la enfermedad, en el apoyo percibido tanto por parte de los amigos como del equipo sanitario. Respecto a éste último, las adolescentes diabéticas perciben menor apoyo. 
Cuanto mayor es el apoyo percibido por parte del equipo de diabetología, el adolescente manifiesta más energía, más bienestar positivo y más bienestar general. No hay asociación con el control metabólico.

Tampoco hay asociaciones estadísticamente significativas entre el apoyo percibido por parte de los amigos y las escalas de bienestar o el control metabólico.

\section{Regresión lineal múltiple}

En los análisis de regresión lineal múltiple de todas las variables consideradas sobre el bienestar, se pone de manifiesto que el sexo es la variable más consistente asociada al mismo. Ser mujer aumenta la puntuación en las escalas de depresión y de ansiedad, mien- tras que ser varón aumenta las de energía, bienestar positivo y bienestar general. En el cuadro se muestran los modelos que mejor se ajustan a los datos. La segunda variable que se asocia de manera consistente a la mayor parte de los indicadores de bienestar es la cohesión familiar percibida por los adolescentes. Las familias más cohesionadas tienen adolescentes diabéticos menos deprimidos, con más energía, más bienestar positivo y más bienestar general. El sexo, la cohesión y una historia de ruptura familiar explican el $43 \%$ de la variabilidad de la depresión medida en la escala de bienestar. El 75\% de la variabilidad del bienestar positivo se explica por el sexo (varón), la cohesión, el apoyo específico a la enfermedad por parte de la familia, una estructura familiar más flexible (menor control) y con

Tabla VI

Regresión múltiple

\begin{tabular}{|c|c|c|c|}
\hline Variable & B & Valor $\mathrm{p}$ & IC $95 \%$ \\
\hline \multicolumn{4}{|c|}{ Depresión $\left(\mathrm{R}^{2}=0,47, \mathrm{R}^{2}\right.$ corregida $\left.=0,43\right)$} \\
\hline Constante & 0,94 & 0,67 & $(-5,4)(3,5)$ \\
\hline Sexo femenino & 3,001 & 0,001 & $(1,2)(4,7)$ \\
\hline Cohesión & $-0,47$ & 0,005 & $(-0,7)(-0,1)$ \\
\hline $\mathrm{H}^{\mathrm{a}}$ Conyugal & 3,66 & 0,01 & $(0,9)(6,4)$ \\
\hline \multicolumn{4}{|c|}{ Ansiedad $\left(R^{2}=0,6 \cdot R^{2}\right.$ corregida $\left.=0,36\right)$} \\
\hline Constante & $-1,84$ & 0,22 & $(-4,8)(1,1)$ \\
\hline Sexo femenino & 4,92 & 0,000 & $(2,9)(6,8)$ \\
\hline \multicolumn{4}{|c|}{ Energía $\left(R^{2}=0,74 . R^{2}\right.$ corregida $\left.=0,52\right)$} \\
\hline Constante & 11,65 & 0,000 & $(9,4)(13,8)$ \\
\hline Sexo femenino & $-3,1$ & 0,000 & $(-4,2)(-2,0)$ \\
\hline Cohesión & 0,34 & 0,001 & $(0,1)(0,5)$ \\
\hline \multicolumn{4}{|c|}{ Bienestar positivo $\left(R^{2}=0,79 . R^{2}\right.$ corregida $\left.=0,75\right)$} \\
\hline Constante & 10,8 & 0,000 & $(6,0)(15,5)$ \\
\hline Sexo femenino & $-4,4$ & 0,000 & $(-5,8)(3,1)$ \\
\hline Cohesión & 0,74 & 0,000 & $(0,4)(1,0)$ \\
\hline CACDI (hijo) & 0,034 & 0,001 & $(0,01)(0,05)$ \\
\hline Social-recreativo & $-0,59$ & 0,007 & $(-1,0)(-0,1)$ \\
\hline Control & $-0,50$ & 0,007 & $(-0,8)(-0,1)$ \\
\hline CACDI (amigos) & 0,06 & 0,01 & $(0,01)(0,1)$ \\
\hline \multicolumn{4}{|c|}{ Bienestar general $\left(R^{2}=0,66 . R^{2}\right.$ corregida $\left.=0,64\right)$} \\
\hline Constante & 58,7 & 0,000 & $(49,7)(67,7)$ \\
\hline Sexo femenino & $-15,7$ & 0,000 & $(-20,3)(-11,1)$ \\
\hline Cohesión & 2,06 & 0,000 & $(1,2)(2,9)$ \\
\hline
\end{tabular}

CACDI: Cuestionario de apoyo al cuidado diabético. 
menos actividades de ocio reglamentadas, así como más apoyo a la enfermedad por parte de los amigos. La cohesión familiar y el sexo explican el $64 \%$ de la variabilidad del bienestar general. El control metabólico no se puede predecir por las variables de bienestar, las características de la dinámica familiar o el apoyo específico a la enfermedad por parte de la familia, los amigos o el equipo médico que hemos considerado.

\section{Discusión}

Hemos hecho un análisis transversal del bienestar emocional en un grupo de adolescentes diabéticos, y su relación con factores del medio familiar y social.

Las diferencias en cuanto al bienestar y género que detectamos en nuestro estudio coinciden en parte con las halladas por la autora de la escala (Bradley 1994). Las adolescentes diabéticas son más propensas a la depresión (Kovacs, Goldston et al. 1997). Además, las mujeres diabéticas manifiestan menor bienestar y calidad de vida (Eiser, Flynn et al. 1992), (Petterson, Lee et al. 1998) (Glasgow, Ruggiero et al. 1997).

Como en nuestro estudio, hay otros, tanto con pacientes diabéticos tipo 1 (Ingersoll y Marrero 1991), (Grey, Boland et al. 1998) como con pacientes tipo 2 (Petterson, Lee $e t$ al. 1998), en los que no se encuentra una asociación entre el control metabólico y ninguna de las medidas de bienestar. Otros trabajos, no obstante, sí hallan una asociación (Guttmann-Bauman, Flaherty et al. 1998) (Dorchy y Olinger 1997, Glasgow, Ruggiero et al. 1997) (Bott, Muhlhauser et al. 1998) (Van der Does, De Neeling et al. 1996). Según Petterson (Petterson, Lee et al. 1998), "la calidad de vida y el control de la glucemia son resultados independientes, existiendo la posibilidad de lograr alguno de ellos a expensas del otro". El buen control metabólico medido por la hemoglobina glicada no es indicador suficiente de éxito del tratamiento en los adolescentes (Grey, Boland et al. 1998). Los pacientes pueden dar mayor importancia a la calidad de vida sobre el buen control metabólico (1996). Por ello, se les debe ayudar a alcanzar el equilibrio mejor para su salud, y tener en cuenta los aspectos relacionados con la calidad de vida a la hora de diseñar los tratamientos (Fallowfield 1996) (Golden 1998) (Ingersoll, Marrero 1991).

Encontramos que las familias con un adolescente diabético son "menos autónomas" y más rígidas en comparación con una familia media española. Para Minuchin (Minuchin, Rosman et al. 1978), estas características son típicas de la llamada por él "familia psicosomática” (aglutinamiento, sobreprotección, rigidez, no resolución de conflictos). Sin embargo, algunos autores (Coyne, Anderson 1988) (Rolland 1994) cuestionan la validez del concepto de "familia psicosomática”. Así, éstas características serían consecuencia o respuestas inevitables a las complicaciones (crisis metabólicas) propias de la evolución de una diabetes juvenil (Coyne y Anderson 1988). Según Rolland (Rolland 1994), "no hay un único patrón familiar que se pueda considerar como saludable o no saludable"; por ejemplo, el grado de cohesión familiar óptimo varía en función de las diferentes fases de los distintos trastornos, del ciclo vital y de la cultura. Por tanto, es un hallazgo que conviene relativizar, no considerarlo en ningún caso como factor causal, y valorar siempre estas características según el contexto de cada familia en particular. La cohesión familiar, en nuestros resultados, es un factor muy ligado al bienestar. 
Nos parece chocante el hallazgo de que a los adolescentes estudiados no parezca satisfacerles que sus familias sean demasiado autónomas, ni que estén excesivamente orientadas a la consecución de objetivos o logros. Estas cualidades disminuyen su bienestar y se asocian a mayor grado de depresión y ansiedad, y menos energía, mientras que un mayor grado de organización parece hacerles sentir mejor. No hemos encontrado en la literatura referencias a estas características de la familia y la calidad de vida. Algunos trabajos previos encuentran una asociación entre el grado de organización, control y autonomía en la familia y la adherencia al tratamiento y adaptación a la enfermedad (Hauser, Jacobson et al. 1985) (Hauser, Jacobson et al. 1990). Los mismos autores no obstante no encuentran asociación entre estas dimensiones y el control metabólico (Jacobson, de Groot et al. 1994).

Una posible explicación sería que, dado que en el tratamiento de la diabetes es necesario mantener una organización y un control (Follansbee 1989), las familias más estructuradas proporcionan un medio más acorde con las necesidades del adolescente diabético.

En nuestro trabajo, el apoyo específico a la enfermedad se asocia a mayor y mejor bienestar de los adolescentes, incluyendo menor depresión y ansiedad. Es particularmente importante en la explicación de la variabilidad del bienestar positivo. Otros trabajos (Hanson, Guire et al. 1992) encuentran que tanto el apoyo general como el específico a la diabetes contribuyen a una mejor adaptación psicosocial de los adolescentes diabéticos. Se ha asociado también a mejor autocuidado (Skinner y Hampson 1998). En el trabajo de Grey (Grey, Boland et al. 1998) sobre calidad de vida, no obstante, no se encuentra ninguna asociación.
Diversos autores defienden la necesidad de implicar a las familias en el manejo de la diabetes, y proceder a una transmisión de la responsabilidad sobre la enfermedad más progresiva, más tardía, para dar tiempo al adolescente a madurar cognitiva y emocionalmente, compartiendo hasta entonces las tareas del tratamiento de la diabetes entre la familia y el paciente (Follansbee 1989) (Greca, Follansbee et al. 1990, Greca 1992, Greca, Auslander et al. 1995). En nuestro trabajo confirmamos el gran valor que los adolescentes prestan a este apoyo, y es congruente también con el hallazgo de que rechacen una independencia excesiva. La meta debe ser conseguir una interdependencia (Follansbee 1989) que fomente la colaboración mutua. Otros autores (Anderson y Brackett et al. 1999) demuestran que esto mejora tanto el manejo de la diabetes de los adolescentes como el control metabólico, y no aumenta el nivel de conflicto intergeneracional. Todo ello apunta a la necesidad de intervenciones estructuradas focalizadas en estos aspectos por parte de los equipos.

De acuerdo con los estudios previos, (Bott, Muhlhauser et al. 1998; Skinner y Hampson 1998, Skinner, John et al. 2000) [47] (Greca 1992, Greca, Auslander et al. 1995), en los que se encuentra que los adolescentes diabéticos valoran el apoyo de los amigos como fundamental en su bienestar emocional, haciéndoles sentirse mejor con su diabetes y mejorando la adherencia al tratamiento, nos hacían pensar que encontraríamos una asociación significativa en nuestro estudio, lo que no se ha confirmado.

Por último, queremos señalar que, salvo en el caso de la energía, no hay un deterioro del bienestar en relación al número de pinchazos de insulina; por tanto, la terapia intensiva en nuestro estudio no se asocia a menor bienestar, confirmando los hallazgos de otros autores (Eiser, Flynn et al. 1992, 1996). 


\section{Bibliografía}

ANDERSON, B.J., BRACKETT, J., HO, J., LAFFEL, LMB. An office-based intervention to maintain parentadolescent teamwork in diabetes management. Diabetes Care 22:713-721, 1999.

BOTT, U., MUHLHAUSER, I., OVERMANN, H., BERGER, M. Validation of a diabetes-specific quality-oflife scale for patients with type 1 diabetes. Diabetes Care 21:757-69, 1998.

BRADLEY, C. The Well-being questionnaire. In Bradley, C. (ed), Handbook of Psychology and Diabetes: Harwood academic publishers, 1994.

BRADLEY, C. Importance of differentiating health status from quality of life. Lancet 357:7-8, 2001.

BRADLEY, C., GAMSU, D.S. Guidelines for encouraging psychological well-being: report of a Working Group of the World Health Organization Regional Office for Europe and International Diabetes Federation European Region St Vincent Declaration Action Programme for Diabetes. Diabet Med 11:510-16, 1994.

BURROUGHS, T.E., HARRIS, M.A., PONTIOUS, S.L., SANTIAGO, J.V. Research on social support in adolescents with IDDM: a critical review. The Diabetes Educator 23:438-448, 1997.

COX, D.J., GONDER-FREDERICK, L. Major developments in behavioral diabetes research. Journal of Consulting and Clinical Psychology 60:628-638, 1992.

COYNE, J.C., ANDERSON, B.J. The "psychosomatic family" reconsidered: diabetes in context. Journal of Marital and Family Therapy 14:113-123, 1988.

DCCT. Reliability and validity of a diabetes quality-oflife measure for the diabetes control and complications trial (DCCT). The DCCT Research Group. Diabetes Care $11: 725-32,1988$.

DCCT. Influence of intensive diabetes treatment on quality-of-life outcomes in the diabetes control and complications trial. Diabetes Care 19:195-203, 1996.

DE SONNAVILLE, J.J., SNOEK, F.J., COLLY, L.P., DEVILLE, W., WIJKEL, D., HEINE, R.J. Well-being and symptoms in relation to insulin therapy in type 2 diabetes. Diabetes Care 21 :919-24, 1998.

DROTAR, D., Relating parent and family functioning to the psychological adjustment of children with chronic health conditions: What have we learned? What do we need to know? Journal of Pediatric Psychology 22: 149$165,1997$.
EISER, C., FLYNN, M., GREEN, E., et al. Quality of life in young adults with type 1 diabetes in relation to demographic and disease variables. Diabet Med 9:375-8, 1992.

FALLOWFIELD, L. Quality of quality of life data. Lancet 348, 1996.

FOLLANSBEE, D.S. Assuming responsibility for diabetes management: what age? What price? Diabetes Educator 15: 347-352, 1989.

GLASGOW, R.E., RUGGIERO, L., EAKIN, E.G., DRYFOOS, J., CHOBANIAN, L. Quality of life and associated characteristics in a large national sample of adults with diabetes. Diabetes Care 20:562-567, 1997.

GOLDEN, M.P. Incorporation of quality-of-life considerations into intensive diabetes management protocols in adolescents. Diabetes Care 21 :885-886, 1998.

GRECA, A.L., FOLLANSBEE, D., SKYLER, J.S. Developmental and behavioral aspects of diabetes management in youngsters. Children's Health Care 19:132-139, 1990.

GRECA, A.M.L. Peer influences in pediatric chronic illness: an update. Journal of Pediatric Psychology 17:775-784, 1992.

GRECA, A.M.L., AUSLANDER, W.F., GRECO, P., SPETTER, D., FISHER, E., SANTIAGO, J. I get by with a little help from my family and friends: adolescent's support for diabetes care. Journal of Pediatric Psychology 20:449-476, 1995.

GREY, M., BOLAND, E.A., DAVIDSON M., LI,, J., TAMBORLANE, W.V. Coping skills training for youth with diabetes mellitus has long-lasting effects on metabolic control and quality of life. J Pediatr 137: 107-13, 2000.

GREY, M., BOLAND, E.A., YU, C., SULLIVANBOLYAI, S., TAMBORLANE, W.V. Personal and family factors associated with quality of life in adolescents with diabetes. Diabetes Care 21 :909-914, 1998.

GUTTMANN-BAUMAN, I., FLAHERTY, B.P., STRUGGER, M., McEVOY, R. Metabolic control and quality of life self-assessment in adolescent with IDDM. Diabetes Care 21: 915-918, 1998.

GUYATT, G.H., FEENY, D.H., PATRICK, D.L. Measuring health-related quality of life. Ann Intern Med 118: 622-629, 1993.

HANSON, C.L., GUIRE, M.D., SCHINKEL, A., HENGGELER, S.W., BUERGHEN, G.A. Comparing social learning and family systems correlates of adaptation in youths with IDDM. Journal of Pediatric Psychology 17:555-572, 1992.

HAUSER, S.T., JACOBSON, A.M., LAVORI, P., et al. Adherence among children and adolescents with insulin- 
dependent diabetes mellitus over a four-year longitudinal follow-up:II. Inmediate and long-term linkages with the family milieu. Journal of Pediatric Psychology 15: 527542, 1990.

HAUSER, S.T., JACOBSON, A.M., WERTLIEB, D., BRINK, S., WENTWORTH, S. The contribution of family environment to perceived competence and illness adjustment in diabetic and acutely ill adolescents. Family Relations 34:99-108, 1985.

HOEY, H., AANSTOOT, H.J., CHIARELLI, F., DANEMAN, D., DANNE, T., DORCHY, H. Good metabolic control is associated with better quality of life in 2101 adolescents with type 1 diabetes. Diabetes Care 24:1923-1928, 2001.

INGERSOLL, G.M., MARRERO, D.G. A modified quality of life measure for youths: psychometric properties. Diabetes Educ 17: 114-118, 1991.

JACOBSON, A.M., DE GROOT, M., SAMSON, J.A. The evaluation of two measures of quality of life in patients with type I and type II diabetes. Diabetes Care 17:267-74, 1994

KATSCHNIG, H., FREEMAN, H., SARTORIUS, N. Prefacio. In Katschnig H, Freeman H, Sartorius N (eds), Calidad de vida en los trastornos mentales: Masson, pp ix, 2000 .

KOVACS, M., GOLDSTON, D., OBROSKY, D.S., BONAR, L.K. Psychiatric disorders in youths with IDDM: drates and risk factors. Diabetes Care 20:36-44, 1997.

MÉNDEZ, F.X., BERMEJO, R., ROS, M.C., HIDALGO, M.D., BELÉNDEZ, M. Elaboración y validación de una batería de tests para niños con diabetes mellitus insulino-dependiente: Proyecto de Investigación del Programa Sectorial de Promoción General del Conocimiento del Ministerio de Educación y Cultura. Referencia: PB961094, 2000.

MINUCHIN, S., ROSMAN, B.L., BAKER, L. Psychosomatic families: Anorexia nervosa in context. Cambridge, MA: Harvard University Press, 1978.

MOOS, R. Family Environment Scale. Palo Alto, CA: Consulting Psychologist Press, 1974.

MOOS, R.H., MOOS, B.S. A typology of family social environments. Fam Process 15:357-371, 1976.

MOOS, R.H., MOOS, B.S., TRICKETT, E.J. Escalas de Clima Social. Madrid: TEA Ediciones, 1995.

PETTERSON, T., LEE, P., HOLLIS, S., YOUNG, B., NEWTON, P., DORNAN, T. Well-being and treatment satisfaction in older people with diabetes. Diabetes Care 21: 930-935, 1998.
POUWER, F., SNOEK, F., PLOEG, H.V.D., ADER, H., HEINE, R. Monitoring of psychological well-being in outpatients with diabetes. Diabetes Care 24: 1929-1935, 2001.

RESNICK, M.D., BEARMAN, P.S., BLUM, R., et al. Protecting adolescents from harm. JAMA 278: 823-832, 1997.

ROLLAND, J.S. Families, illness and disability. An integrative treatment model. New York: Basic Books, 1994.

SKINNER, T.C., HAMPSON, S.E. Social support and personal models of diabetesin relation to self-care and well-being inadolescents with type I diabetes mellitus. $J$ Adolesc 21 :703-715, 1998.

SKINNER, T.C., HAMPSON, S.E. Personal models of diabetes in relation to self-care, well-being, and glycemic control. A prospective study in adolescence. Diabetes Care 24:828-833, 2001.

SKINNER, T.C., JOHN, M., HAMPSON, S.E. Social support and personal models of diabetes as predictors of self-care and well-being: a longitudinal study of adolescents with diabetes. J Pediatr Psychol 25: 257-267, 2000.

STEWART, A.L., GREENFIELD, S., HAYS, R.D., et $a l$. Functional status and well-being of patients with chronic conditions. Results from the Medical Outcomes Study. Jama 262: 907-13, 1989.

VAN DER DOES, F.E., DE NEELING, J.N., SNOEK F.J., et al. Symptoms and well-being in relation to glycemic control in type II diabetes. Diabetes Care 19:204-10, 1996.

VARNI, J.W., BABANI, L., WALLANDER, J.L., ROE, T.F., FRASIER, S.D. Social support and self-esteem effects on psychological adjustment in children and adolescents with insulin dependent diabetes mellitus. Child \& Family Beharior Therapy 11: 1-17, 1989.

WU, S.Y., SAINFORT, F., TOMAR, R.H., et al. Development and application of a model to estimate the impact of type 1 diabetes on health-related quality of life. Diabetes Care 21:725-31, 1998.

Address of correspondence:

Dra. C. de Dios

e-mail: Cdedios@inicia.es

Servicio de Psiquiatría

Hospital Universitario La Paz

$\mathrm{P}^{\circ}$ de la Castellana, 261

28046 Madrid

ESPAÑA 